\title{
Hydrazinopeptide Motifs Synthesized via the Ugi Reaction: an Insight into the Secondary Structure
}

\author{
Mikhail Krasavin, ${ }^{* a}$ Ekaterina Bushkova, ${ }^{a}$ Vladislav Parchinsky, ${ }^{b}$ Alexei Shumsky ${ }^{b}$ \\ ${ }^{a}$ Science and Education Center “Innovative Research”, Yaroslavl State Pedagogical University,
} 150000, Russia

${ }^{b}$ Chemical Diversity Research Institute, 2a Rabochaya St., Khimki, Moscow Reg., 141400, Russia

Phone: +7(495)995-4944. Fax: +7(495)626-9780. E-mail: myk@chemdiv.com

\begin{abstract}
A number of $N^{(\alpha)}$-alkyl, $N^{(\beta)}$-acyl hydrazines have been synthesized via the Ugi reaction of $\mathrm{N}$-acylhydrazones with isocyanide and TFA. TFA acted as a 'silent partner' and becomes removed upon basic workup of the reaction. These compounds have been efficiently modified further via resuctive amination to produce $N^{(\alpha)}, N^{(\alpha)}$-bisalkyl, $N^{(\beta)}$-acyl hydrazines. The two groups of the novel hydrazinopeptide motifs have been shown to display two different secondary structure patterns by simple ${ }^{1} \mathrm{H}$ NMR experiments. These observations were confirmed by X-ray crystallographic analysis. Combining hydrazone and carboxylic acid moieties in one reaction precursor offers an opportunity for an 'intramolecular' hydrazino-Ugi reaction, which was demonstrated in this work as well.
\end{abstract}

Key words: multicomponent reaction, hydrazones, substituent effects, peptidomimetics, secondary structure.

The four-component reaction of an amine, a carbonyl compound, a carboxylic acid and an isocyanide (known as the Ugi reaction ${ }^{1}$ ) delivers medicinally relevant ${ }^{2}$ dipeptoid compounds in remarkably efficient, atom-economic manner (the only by-product being the condensation water from the initial imine formation). The reaction has gone a long evolutionary path since its discovery in 1959. The common theme in developing novel isocyanide-based multicomponent reactions (IMCRs) has been the replacement of one or more reaction partners in the 'Ugi foursome' with various surrogates. The most developed strategy to-date is the use of noncarboxylate nucleophiles to intercept the isocyanide interacting with the iminium species. ${ }^{3}$ However, finding workable surrogates for the iminium component (formation of which from the amine and the carbonyl compounds under acidic catalysis triggers the Ugi reaction) appears more challenging as one find only a handful of examples of this approach in the literature. Among them are the successful uses of various hydrazones, ${ }^{4}$ oximes, ${ }^{5}$ and more recently 
published methodologies involving $N$-acylazinium ${ }^{6}$ and $N$-fluoropyridinium ${ }^{7}$ salts as less obvious substitutes for the iminium reactive partner for an isocyanide.

We have recently modified the early methodology by $\mathrm{Ugi}^{4 \mathrm{~b}}$ and employed trifluoroacetic acid as the carboxylic acid partner in the reaction of hydrazones 1 with isocyanides 2 . The initially formed 'hydrazino-Ugi' products $\mathbf{3}$ contain a labile trifluoroacetate group that is easily removed under mild basic hydrolysis of the isolated 3 or in situ. The resulting $N^{(\alpha)}$-alkyl, $N^{(\beta)}$-acyl hydrazines 4 represent hydrazinopeptide-like ${ }^{8}$ structures and also contain a reactive $N^{(\alpha)}$ atom that can be used for further derivatization of the newly formed hydrazinopeptide backbone, e. g., through a second Ugi reaction (Scheme 1). ${ }^{9}$ Hydrazinopeptides represent a less studied class of peptidomimetics useful in preparing more proteolytically stable ${ }^{10}$ analogs of natural bioactive peptides with preserved biological activity. ${ }^{11}$ In addition, these motifs are quite appealing in terms of their secondary structure as they are known ${ }^{12}$ do adopt a unique secondary structure $-\mathrm{a}$ 'hydrazino turn' owing to the bifurcated hydrogen bond involving the $s p^{3}$-hydridized $N^{(\alpha)}$ atom similar to the natural peptide $\beta$-turn (Figure 1).

Herein we report on the synthesis of an extended set of hydrazinopeptide units 4, their modification at the $N^{(\alpha)}$ atom via reductive alkylation. The propensity of such structures to yield intriguing secondary structure patterns also prompted us to investigate the latter by means of NMR spectroscopy and X-ray crystallography to reveal marked regularity that we disclose in this article.

Thirteen hydrazinopeptide motifs $\mathbf{4 a - m}$ were synthesized according to one-pot procedure, with mild basic workup of the reaction mixture leading to clean and complete removal of the trifluoroacetyl group (Scheme 1). The isolated yields were moderate to good (Table 1) and the identity of the compounds was consistent with the characterization data. Interestingly, compounds 4i-j are essentially the racemic Boc-protected hydrazine analogs of valine amides. ${ }^{13}$ It should be noted that the trifluoroacetyl hydrazines $\mathbf{3}$ are, in principle, stable to isolation, as demonstrated by the isolation and characterization of a representative compound $3 \mathbf{i}$ (en route to 4i). The choice of dioxane as the medium for the hydrazino-Ugi reaction is important as the same reaction carried out in methanol (often the solvent 'of choice' for a number of Ugi reactions ${ }^{14}$ ) leads to a more complex mixture of products as shown for two reactions presented in the Scheme 2. Without the basic workup, the reaction mixtures were analyzed by LC MS to reveal the presence of equal amounts of the expected hydrazine-Ugi product $\mathbf{3}$ and de-trifluoroacetylated product 4. In addition, substantial amounts of the methyl ester 5 were present in the reaction mixtures, these were isolated chromatographically in the yields indicated and characterized. 
Formation of the products 4 and 5 in methanol can be rationalized by possible interference of the nucleophilic solvent molecule with the course of the reaction, as indicated in the Scheme 3.

If two reactive partners for the Ugi process are combined within the structure of a single reactant, a ring-forming process can occur (as has been shown for various keto and aldehydo acids as substrates for a four-center three-component Ugi reaction ${ }^{15}$ ). We designed a precursor 6 containing both the hydrazone and the carboxylic acid moieties, that was synthesized in four straightforward chemical operations (involving one purification). In analogy to the previously reported preparation of aza- $\beta$-lactams from $\alpha$-hydrazino acids via the Ugi reaction, ${ }^{16} 6$ produced $N$-monoalkylated tetrahydropyridazine-3,6-dione 7 in good yield, upon 'intramolecular' Ugi reaction with tert-butyl isocyanide in isopropanol (Scheme 4). Notably, when the same reaction was performed in methanol, the methyl ester 8 was identified by LC MS as the main reaction product (though we failed to isolate it from the reaction mixture). Its formation, again, can be rationalized by methanol (which is more nucleophilic than isopropanol) interfering with the course of the 'intramolecular' Ugi reaction (Scheme 5).

The $N^{(\alpha)}$ atom of the compounds $\mathbf{4}$ is a potential reactive center and can be used for introduction of further diversity off the hydrazine moiety, as was demonstrated by its participation in the Ugi reaction (Scheme 1). ${ }^{9}$ We turned to explore its reactivity in reductive alkylation reactions. Although we found reductive alkylation unfeasible with aromatic or heteroaromatic aldehydes (in a range of solvents, temperatures and with various reductants), a set of ten $N^{(\alpha)}, N^{(\alpha)}$-bisalkyl, $N^{(\beta)}$-acyl hydrazines 9a-k was successfully prepared in good to excellent yields (Table 2) using isovaleric and butyric aldehydes, as shown in the Scheme 6.

Having prepared two sets of hydrazinopeptide fragments (4 and 9) we proceeded to study their secondary structure. By comparing changes in ${ }^{1} \mathrm{H}$ NMR chemical shifts of the protons in $\mathbf{4}$ and 9 'suspected' of participation in intramolecular hydrogen bonding with the same changes in reference fragments $\mathbf{1 0}$ and $\mathbf{1 1}$ (Figure 2) ${ }^{18}$ where no such bonding is possible, upon solvent change from $d_{6}$-DMSO to $d$-chloroform, one can pinpoint those protons that are indeed involved in intramolecular H-bond. ${ }^{17}$ Indeed, as can be seen from the Table 3, $d_{6}$-DMSO as solvent capable of accepting hydrogen bond from the solute, causes downfield shift of the acidic, amidetype protons $\mathrm{H}^{1}$ and $\mathrm{H}^{2}$ in the reference fragments $\mathbf{1 0}$ and $\mathbf{1 1} .{ }^{19}$ Roughly the same downfield shift of ${ }^{1} \mathrm{H}$ NMR signals was observed $N^{(\beta)}-\mathrm{H}^{1}$ protons of $\mathbf{4}$ but not for the terminal amide protons $\mathrm{H}^{2}$ which were significantly less sensitive to the solvent change than the respective $\mathrm{H}^{2}$ protons in $\mathbf{1 1}$. This can be explained only by involvement of the latter in intramolecular H-bond. The situation reverses for the compounds $\mathbf{9}$; and this observation prompted us to conclude that introduction of 
the second alkyl at $N^{(\alpha)}$ makes an alternative hydrogen bonding pattern (involving $\mathrm{H}^{1}$ but not $\mathrm{H}^{2}$ this time) more favorable. To our delight, the above conclusions were fully confirmed by singlecrystal X-ray analysis ${ }^{20}$ of compounds $4 \mathbf{i}$ and $\mathbf{9 h}$, representative of each set of the hydrazinopeptide units studied. Indeed, 4i adopted the typical hydrazine turn secondary structure while in $\mathbf{9 h}$ this structural bias was not observed (Figure 3). ${ }^{21}$ These observations are expected to be of importance in selection of appropriate hydrazinopeptide inserts for the natural peptide analog design.

In conclusion, we have reported on the application of the Ugi reaction of hydrazones toward simple and efficient preparation of racemic hydrazinopeptide units 4. An 'intramolecular' version of the same reaction was developed using the hydrazone 6 containing carboxylic acid side chain. Modification of $\mathbf{4}$ at $N^{(\alpha)}$ via reductive alkylation by aliphatic aldehydes produced compounds 9. As demonstrated by simple ${ }^{1} \mathrm{H}$ NMR experiments in solvents with different hydrogen bond acceptor ability and confirmed by crystallographic analysis, compounds $\mathbf{4}$ and $\mathbf{9}$ consistently displayed different secondary structure patterns.

All reactions were run in oven-dried glassware in atmosphere of nitrogen. Melting points were measured with a Buchi B-520 melting point apparatus and were not corrected. Analytical thin-layer chromatography was carried out on EM Separations Technology $\mathrm{F}_{254}$ silica gel plates. Compounds were visualized with short-wavelength UV light. ${ }^{1} \mathrm{H}$ NMR and ${ }^{13} \mathrm{C}$ NMR spectra were recorded on Bruker DPX-300 spectrometers in DMSO- $d_{6}$ using TMS as an internal standard. LCMS analyses were obtained on a PE SCIEX API 150EX mass spectrometer following separation on a Shimadzu LC-10AD liquid chromatography system equipped with Shimadzu SP D-10A UV-Vis detector $(254 \mathrm{~nm})$ and Sedex 75 ELSD detector. Elemental analyses were obtained at Research Institute for Chemical Crop Protection (Moscow, Russia) using Carlo Erba Strumentazione 1106 analyzer. All solvents and reagents were obtained from commercial sources and used without purification.

\section{2-Cyclohexyl- $N^{1}$-(2-methoxyethyl)-2-[2-(4-pyridylcarbonyl)hydrazino]acetamide Typical Procedure for the Synthesis of Compounds 4a-m}

(4a);

N'-(Cyclohexylmethylene)isonicotinohydrazide $(600 \mathrm{mg}, 2.6 \mathrm{mmol})$ was dissolved in anhydrous dioxane $(10 \mathrm{~mL})$. 2-Methoxyethyl isocyanide $(320 \mathrm{~mL}, 3.9 \mathrm{mmol})$ was added followed by trifluoroacetic acid $(193 \mathrm{~mL}, 2.6 \mathrm{mmol})$. The reaction was stirred at $\mathrm{r}$. t. overnight and then diluted with $10 \%$ aq. $\mathrm{K}_{2} \mathrm{CO}_{3}(25 \mathrm{~mL})$ and stirred for $1 \mathrm{~h}$. The solution was extracted with chloroform ( $3 \times 50 \mathrm{~mL}$ ), the extracts were dried over anydrous $\mathrm{MgSO}_{4}$, filtered, and concentrated under reduced pressure. Chromatography on silica gel using $10 \rightarrow 65 \%$ ethyl acetate in hexanes as eluent provided $\mathbf{4 a}(450 \mathrm{mg}, 52 \%)$.

White solid; mp $132-134{ }^{\circ} \mathrm{C}$. 
${ }^{1} \mathrm{H}$ NMR (DMSO- $\left.d_{6}, 300 \mathrm{~K}, \mathrm{ppm}\right): \delta 0.98 \ldots 1.34(\mathrm{~m}, 5 \mathrm{H}), 1.50 \ldots 1.85(\mathrm{~m}, 6 \mathrm{H}), 2.93(\mathrm{~d}, J=2.8$ $\mathrm{Hz}, 1 \mathrm{H}), 3.14 \ldots 3.43(\mathrm{~m}, 7 \mathrm{H}), 5.24(\mathrm{bs}, 1 \mathrm{H}), 7.62(\mathrm{~m}, 3 \mathrm{H}), 8.66(\mathrm{~d}, J=1.8 \mathrm{~Hz}, 2 \mathrm{H}), 9.77(\mathrm{bs}, 1 \mathrm{H})$.

${ }^{13} \mathrm{C}$ NMR (DMSO- $\left.d_{6}, 300 \mathrm{~K}, \mathrm{ppm}\right): \delta 25.8,28.6,28.9,38.1,38.6,57.8,68.6,70.5,121.1,140.2$, $150.1,163.5,171.4$.

Anal. Calcd for $\mathrm{C}_{17} \mathrm{H}_{26} \mathrm{~N}_{4} \mathrm{O}_{3}$ : C,61.06; H,7.84; N,16.75. Found: C,61.16; H,7.92; N,16.77.

\section{2-Cyclohexyl- $N^{1}$-(2-methoxyethyl)-2-2-[2-(4-methylphenyl)acetyl]hydrazinoacetamide (4b)}

White solid; mp $108-110^{\circ} \mathrm{C}$.

${ }^{1} \mathrm{H}$ NMR (DMSO-d $\left.6,300 \mathrm{~K}, \mathrm{ppm}\right): \delta 0.99 \ldots 1.19(\mathrm{~m}, 5 \mathrm{H}), 1.48 \ldots 1.71(\mathrm{~m}, 6 \mathrm{H}), 2.25(\mathrm{~s}, 3 \mathrm{H}), 3.03$ $(\mathrm{d}, J=2.9 \mathrm{~Hz} 1 \mathrm{H}), 3.10 \ldots 3.27(\mathrm{~m}, 9 \mathrm{H}), 4.98$ (bs, 1H), 7.08 (m, 4H), 7.86 (bs, 1H), 9.33 (bs, 1H).

${ }^{13} \mathrm{C}$ NMR (DMSO-d $\left.d_{6}, 300 \mathrm{~K}, \mathrm{ppm}\right): \delta 20.5,25.8,28.4,28.9,38.0,39.3,57.8,69.0,70.4,128.7$, $132.9,135.3,169.2,171.4$.

Anal. Calcd for $\mathrm{C}_{20} \mathrm{H}_{31} \mathrm{~N}_{3} \mathrm{O}_{3}$ : C,66.45; H, 8.64; N, 11.62. Found: C,66.51; H, 8.67; N, 11.77.

\section{2-Cyclohexyl- $N^{1}$-(2-methoxyethyl)-2-[2-(2-pyrazinylcarbonyl)hydrazino]acetamide (4c)}

White solid; mp $108-110^{\circ} \mathrm{C}$.

${ }^{1} \mathrm{H}$ NMR (DMSO-d $\left.d_{6}, 300 \mathrm{~K}, \mathrm{ppm}\right): \delta 1.06 \ldots 1.30(\mathrm{~m}, 5 \mathrm{H}), 1.53 \ldots 1.85(\mathrm{~m}, 6 \mathrm{H}), 3.21(\mathrm{~s}, 3 \mathrm{H})$, $3.22 \ldots 3.40(\mathrm{~m}, 4 \mathrm{H}), 5.23$ (bs, 1H), 7.73 (bs, 1H), 8.61 (bs, 1H), 8.81 (bs, 1H), 9.12 (bs, 1H), 9.67 (bs, $1 \mathrm{H})$.

${ }^{13} \mathrm{C}$ NMR (DMSO-d $\left.d_{6}, 300 \mathrm{~K}, \mathrm{ppm}\right): \delta 25.9,28.5,29.0,38.0,38.7,57.8,68.0,70.5,143.3,143.4$, $144.4,147.5,161.1,171.6$.

Anal. Calcd for $\mathrm{C}_{16} \mathrm{H}_{25} \mathrm{~N}_{5} \mathrm{O}_{3}$ : C,57.30; H, 7.51; N, 20.88. Found: C,57.39; H, 7.64; N, 21.06.

\section{$N^{1}$-(2-Methoxyethyl)-2-[2-(4-pyridylcarbonyl)hydrazino]pentanamide (4d)}

White solid, mp $123-125^{\circ} \mathrm{C}$.

${ }^{1} \mathrm{H}$ NMR (DMSO- $\left.d_{6}, 300 \mathrm{~K}, \mathrm{ppm}\right): \delta 0.90(\mathrm{t},, J=2.3 \mathrm{~Hz}, 3 \mathrm{H}), 1.40(\mathrm{~m}, 2 \mathrm{H}), 1.58(\mathrm{~m}, 2 \mathrm{H}), 2.98$ (bs, 1H), 3.18...3.49 (m, 7H), $5.46(\mathrm{bs}, 1 \mathrm{H}), 7.65(\mathrm{~d}, J=1.8 \mathrm{~Hz}, 2 \mathrm{H}), 7.78(\mathrm{bs}, 1 \mathrm{H}), 8.68(\mathrm{~d}, J=$ $1.8 \mathrm{~Hz}, 2 \mathrm{H}), 9.83$ (bs, $1 \mathrm{H})$.

${ }^{13} \mathrm{C}$ NMR (DMSO-d $\left.6,300 \mathrm{~K}, \mathrm{ppm}\right): \delta 13.9,18.4,33.1,26.4,57.8,63.3,70.5,121.2,140.4$, $149.9,163.7,172.2$.

Anal. Calcd for $\mathrm{C}_{14} \mathrm{H}_{22} \mathrm{~N}_{4} \mathrm{O}_{3}$ : C,57.13; H,7.53; N,19.03. Found: C,57.33; H,7.50; N,18.96. 


\section{$N^{1}$-benzyl-2-[2-(4-pyridylcarbonyl)hydrazino]pentanamide (4e)}

Beige solid, mp $147-149^{\circ} \mathrm{C}$.

${ }^{1} \mathrm{H}$ NMR (DMSO- $\left.d_{6}, 300 \mathrm{~K}, \mathrm{ppm}\right): \delta 0.91(\mathrm{t},, J=6.7 \mathrm{~Hz}, 3 \mathrm{H}), 1.42(\mathrm{~m}, 2 \mathrm{H}), 1.63(\mathrm{~m}, 2 \mathrm{H}), 3.52$ $(\mathrm{t}, J=3.5 \mathrm{~Hz}, 1 \mathrm{H}), 4.32\left(\mathrm{ddd}, J_{1}=13.9 \mathrm{~Hz}, J_{2}=5.2 \mathrm{~Hz}, J_{3}=4.0 \mathrm{~Hz}, 2 \mathrm{H}\right), 5.23(\mathrm{bs}, 1 \mathrm{H})$, 7.15...7.30 (m, 5H), $7.63(\mathrm{~d}, J=3.4 \mathrm{~Hz}, 2 \mathrm{H}), 8.15(\mathrm{bs}, 1 \mathrm{H}), 8.68$ (d, $J=3.4 \mathrm{~Hz}, 2 \mathrm{H}), 9.89$ (bs, $1 \mathrm{H})$;

${ }^{13} \mathrm{C}$ NMR (DMSO- $\left.d_{6}, 300 \mathrm{~K}, \mathrm{ppm}\right): \delta 13.9,18.5,33.9,14.9,63.3,121.0,126.6,127.0,128.1$, $139.3,140.0,150.0,163.7,172.2$.

Anal. Calcd for $\mathrm{C}_{18} \mathrm{H}_{22} \mathrm{~N}_{4} \mathrm{O}_{2}$ : C,66.24; H,6.79; N,17.16. Found: C,66.07; H,6.88; N,17.27.

\section{$N^{1}$-Benzyl-2-cyclohexyl-2-[2-(2-pyrazinylcarbonyl)hydrazino]acetamide (4f).}

Grey solid, mp $163-166^{\circ} \mathrm{C}$.

${ }^{1} \mathrm{H}$ NMR (DMSO-d $\left.d_{6}, 300 \mathrm{~K}, \mathrm{ppm}\right): \delta 1.03 \ldots 1.33(\mathrm{~m}, 5 \mathrm{H}), 1.55 \ldots 1.88(\mathrm{~m}, 6 \mathrm{H}), 3.41(\mathrm{~d}, J=5.1$ $\mathrm{Hz}, 1 \mathrm{H}), 4.32$ (ddd, $\left.J_{1}=18.7 \mathrm{~Hz}, J_{2}=6.0 \mathrm{~Hz}, J_{3}=5,2 \mathrm{~Hz}, 2 \mathrm{H}\right), 5.29(\mathrm{bs}, 12 \mathrm{H}), 7.12 \ldots 7.28(\mathrm{~m}$, $5 \mathrm{H}), 8.19$ (bs, 1H), 8.65 (d, $J=1.8 \mathrm{~Hz} 1 \mathrm{H}), 8.82$ (d, $J=1.8 \mathrm{~Hz} 1 \mathrm{H}), 9.10(\mathrm{~s}, 1 \mathrm{H}), 9.79$ (bs, 1H).

${ }^{13} \mathrm{C}$ NMR (DMSO- $\left.d_{6}, 300 \mathrm{~K}, \mathrm{ppm}\right): \delta$ 25.8, 25.9, 28.6 29.1, 41.9, 68.0, 126.5, 127.1, 127.9, $139.4,143.2,143.3,144.3,147.5,160.9,171.7$.

Anal. Calcd for $\mathrm{C}_{20} \mathrm{H}_{25} \mathrm{~N}_{5} \mathrm{O}_{2}$ : C, 65.37; H, 6.86; N, 19.06. Found: C, 65.33; H, 6.91; N, 18.92.

\section{$N^{1}$-Benzyl-2-cyclohexyl-2-[2-(4-pyridylcarbonyl)hydrazino]acetamide (4g)}

Grey solid, mp $115-116{ }^{\circ} \mathrm{C}$.

${ }^{1} \mathrm{H}$ NMR (DMSO- $\left.d_{6}, 300 \mathrm{~K}, \mathrm{ppm}\right): \delta 1.09 \ldots 1.30(\mathrm{~m}, 5 \mathrm{H}), 1.55 \ldots 1.87(\mathrm{~m}, 6 \mathrm{H}), 3.34(\mathrm{~d}, J=3.9$ $\mathrm{Hz}, 1 \mathrm{H}), 4,32\left(\mathrm{ddd}, J_{1}=22.1 \mathrm{~Hz}, J_{2}=6.6 \mathrm{~Hz}, J_{3}=5.8 \mathrm{~Hz}, 2 \mathrm{H}\right), 5.30(\mathrm{bs}, 1 \mathrm{H}), 7.24(\mathrm{~m}, 5 \mathrm{H}), 7.62$ $(\mathrm{d}, J=2.6 \mathrm{~Hz}, 2 \mathrm{H}), 8.10$ (bs, 1H), 8.67 (d, $J=2.6 \mathrm{~Hz}, 2 \mathrm{H}), 9.84$ (bs, 1H).

${ }^{13} \mathrm{C}$ NMR (DMSO- $\left.d_{6}, 300 \mathrm{~K}, \mathrm{ppm}\right): \delta 25.7,27.4,28.9,29.1,41.9,68.6,121.1,126.6,127.1$, $128.0,139.4,140.0,150.1,163.3,171.5$.

Anal. Calcd for $\mathrm{C}_{21} \mathrm{H}_{26} \mathrm{~N}_{4} \mathrm{O}_{2}$ : C,68.83; H,7.15; N,15.29. Found: C,68.80; H,7.25; N,15.38.

\section{N-Cyclohexyl-2-\{2-[(4-methylphenyl)acetyl]hydrazino\}butanamide (4h)}

White solid, mp $150-152{ }^{\circ} \mathrm{C}$. 
${ }^{1} \mathrm{H}$ NMR (DMSO- $\left.d_{6}, 300 \mathrm{~K}, \mathrm{ppm}\right): \delta 0.83(\mathrm{t}, J=7.5 \mathrm{~Hz}, 3 \mathrm{H}), 0.89 \ldots 1.28(\mathrm{~m}, 5 \mathrm{H}), 1.44 \ldots 1.70(\mathrm{~m}$, $7 \mathrm{H}), 2.25(\mathrm{~s}, 3 \mathrm{H}), 3.13(\mathrm{~m}, 1 \mathrm{H}), 3.44(\mathrm{~m}, 1 \mathrm{H}), 5.0(\mathrm{bs}, 1 \mathrm{H}), 7.06(\mathrm{~d}, J=8.2 \mathrm{~Hz}, 2 \mathrm{H}), 7.1$ (d, J=8.2 $\mathrm{Hz}, 2 \mathrm{H}), 7.76(\mathrm{~d}, J=8.0 \mathrm{~Hz}, 1 \mathrm{H}), 9.34(\mathrm{~d}, J=3.3 \mathrm{~Hz}, 1 \mathrm{H})$.

${ }^{13} \mathrm{C}$ NMR (DMSO-d $\left.6,300 \mathrm{~K}, \mathrm{ppm}\right): \delta 9.8,20.5,23.9,24,4$ 25.2, 32.0, 32.3, 47.2, 65.0, 128.6, $128.7,132.8,135.3,169.4,170.7$.

Anal. Calcd for $\mathrm{C}_{19} \mathrm{H}_{29} \mathrm{~N}_{3} \mathrm{O}_{2}: \mathrm{C}, 68.85 ; \mathrm{H}, 8.82 ; \mathrm{N}, 12.68$. Found: $\mathrm{C}, 68.95 ; \mathrm{H}, 8.91 ; \mathrm{N}, 12.74$.

\section{$N^{1}$-(tert-Butyl)-2-2-[2-(4-methoxyphenoxy)acetyl]hydrazino-4-methylpentanamide (4i)}

White solid, mp $173^{\circ} \mathrm{C}$ (decomp.).

${ }^{1} \mathrm{H}$ NMR (DMSO- $\left.d_{6}, 300 \mathrm{~K}, \mathrm{ppm}\right): \delta 0.9$ (d, $\left.J=5.9 \mathrm{~Hz}, 6 \mathrm{H}\right), 1.28(\mathrm{~s}, 9 \mathrm{H}), 1.40(\mathrm{~m}, 2 \mathrm{H}), 1.80$ $(\mathrm{m}, 1 \mathrm{H}), 3.30(\mathrm{t}, J=6.4 \mathrm{~Hz}, 1 \mathrm{H}), 3.72(\mathrm{~s}, 3 \mathrm{H}), 4.44(\mathrm{bs}, 2 \mathrm{H}), 4.85(\mathrm{bs}, 1 \mathrm{H}), 6.86(\mathrm{~d}, J=9.3,2 \mathrm{H})$, $6.90(\mathrm{~d}, J=9.3,2 \mathrm{H}), 7.32(\mathrm{bs}, 1 \mathrm{H}), 9.02(\mathrm{bs}, 1 \mathrm{H})$.

${ }^{13} \mathrm{C}$ NMR (DMSO-d 6 , $\left.300 \mathrm{~K}, \mathrm{ppm}\right): \delta 22.3,23.0,24.2,28.4,40.5,49.6,55.9,62.9,67.2,114.5$, $115.7,151.6,153.9,166.7,172.1$.

Anal. Calcd for $\mathrm{C}_{19} \mathrm{H}_{31} \mathrm{~N}_{3} \mathrm{O}_{4}$ : C, 62.44; H, 8.55; N, 11.50. Found: C, 62.49; H, 8.64; N, 11.30.

\section{$N^{1}$-(tert-butyl)-2-[2-[2-(4-methoxyphenoxy)acetyl]-1-(2,2,2-trifluoroacetyl)hydrazino]-4-} methylpentanamide (3i)

The compound was prepared according to the same procedure as $\mathbf{4 i}$ but the basic workup was omitted.

Off-white solid, mp $148^{\circ} \mathrm{C}$ (decomp.).

${ }^{1} \mathrm{H}$ NMR (DMSO-d $\left., 363 \mathrm{~K}, \mathrm{ppm}\right): \delta 0.88(\mathrm{~d}, J=6.4 \mathrm{~Hz}, 6 \mathrm{H}), 1.27(\mathrm{~s}, 9 \mathrm{H}), 1.47 \ldots 1.74(\mathrm{~m}, 3 \mathrm{H})$, $3.72(\mathrm{~s}, 3 \mathrm{H}), 4.48(\mathrm{t}, J=7.1 \mathrm{~Hz}, 1 \mathrm{H}), 4.64(\mathrm{~s}, 2 \mathrm{H}), 6.87$ (d, J=9.2 Hz, 2H), 6.94 (d, J = 9.2 Hz, 2H), 7.75 (bs, 1H), 10.4 (bs, 1H).

${ }^{13} \mathrm{C}$ NMR (DMSO-d $\left., 363 \mathrm{~K}, \mathrm{ppm}\right): \delta 22.1,22.3,24.2,28.2,37.1,50.4,55.8,62.1,67.0,115.0$, $115.5(J=288.5 \mathrm{~Hz}), 116.2,152.0,154.5,157.4(J=35.8), 167.8,169.0$.

Anal. Calcd for $\mathrm{C}_{21} \mathrm{H}_{30} \mathrm{~F}_{3} \mathrm{~N}_{3} \mathrm{O}_{5}$ : C, 54.66; H, 6.55; N, 9.11. Found C, 54.60; H, 6.43; N, 8.97.

\section{tert-Butyl 2-(1-[(tert-butylamino)carbonyl]-2-methylpropyl-1-hydrazinecarboxylate (4j)}

White solid, mp $172-174{ }^{\circ} \mathrm{C}$.

${ }^{1} \mathrm{H}$ NMR (DMSO-d $\left.6,300 \mathrm{~K}, \mathrm{ppm}\right): \delta 0.84(\mathrm{~d}, J=7.0 \mathrm{~Hz} 3 \mathrm{H}), 0.91(\mathrm{~d}, J=7.0 \mathrm{~Hz} 3 \mathrm{H}) 1.25(\mathrm{~s}$, 9H), 1.39 (s, 9H), $1.86(\mathrm{~m}, 1 \mathrm{H}), 2.92$ (d, , J = 3.8 Hz 1H ), 4.73 (s, 1H), 7.63 (bs, 1H), 8.20 (bs, $1 \mathrm{H})$. 
${ }^{13} \mathrm{C}$ NMR (DMSO- $\left.d_{6}, 300 \mathrm{~K}, \mathrm{ppm}\right): \delta 18.2,18.9,28.2,28.3,29.5,49.6,70.1,78.3,156.2,170.8$. Anal. Calcd for $\mathrm{C}_{14} \mathrm{H}_{29} \mathrm{~N}_{3} \mathrm{O}_{3}$ : C,58.51; H, 10.17; N, 14.62. Found: C,58.43; H, 10.07; N, 14.77.

tert-butyl 2-(1-[(4-methoxybenzyl)amino]carbonyl-2-methylpropyl)-1-hydrazinecarboxylate (4k)

White solid, mp $180{ }^{\circ} \mathrm{C}$ (decomp.).

${ }^{1} \mathrm{H}$ NMR (DMSO- $\left.d_{6}, 300 \mathrm{~K}, \mathrm{ppm}\right): \delta 0.93(\mathrm{t}, J=6.9 \mathrm{~Hz}, 6 \mathrm{H}), 1.40(\mathrm{~s}, 9 \mathrm{H}), 1.95(\mathrm{~m}, 1 \mathrm{H}), 3.14$ (bs, $1 \mathrm{H}), 3.75(\mathrm{~s}, 3 \mathrm{H}), 4.25\left(\mathrm{ddd}, J_{1}=36.5 \mathrm{~Hz}, J_{2}=5.0 \mathrm{~Hz}, J_{3}=5,3 \mathrm{~Hz}, 2 \mathrm{H}\right), 4.50(\mathrm{~s}, 1 \mathrm{H}), 6.86$ $(\mathrm{d}, J=8.2,2 \mathrm{H}), 7.21(\mathrm{~d}, J=8.2,2 \mathrm{H}), 8.11(\mathrm{bs}, 1 \mathrm{H}), 8.22(\mathrm{bs}, 1 \mathrm{H})$.

${ }^{13} \mathrm{C}$ NMR (DMSO- $\left.d_{6}, 300 \mathrm{~K}, \mathrm{ppm}\right): \delta 18.3,18.9,28.2,29.7,41.7,55.2,69.8,78.7,113.9,128.5$, $131.6,156.2,158.4,171.6$

Anal. Calcd for $\mathrm{C}_{18} \mathrm{H}_{29} \mathrm{~N}_{3} \mathrm{O}_{4}: \mathrm{C}, 61.52 ; \mathrm{H}, 8.32 ; \mathrm{N}, 11.96$. Found $\mathrm{C}, 61.57 ; \mathrm{H}, 8.02 ; \mathrm{N}, 12.13$.

\section{2-(2-Acetylhydrazino)-2-cyclohexyl- $N^{1}$-(2-methoxybenzyl)acetamide (4l)}

White solid, mp 166-168 ${ }^{\circ} \mathrm{C}$.

${ }^{1} \mathrm{H}$ NMR (DMSO-d $\left.6,300 \mathrm{~K}, \mathrm{ppm}\right): \delta 1.03 \ldots 1.29(\mathrm{~m}, 5 \mathrm{H}), 1.53 \ldots 1.92(\mathrm{~m}, 9 \mathrm{H}), 3.14(\mathrm{~d},, J=1.6$ $\mathrm{Hz} 1 \mathrm{H}), 3.81(\mathrm{~s}, 3 \mathrm{H}), 4.30(\mathrm{~m}, 2 \mathrm{H}), 4.87(\mathrm{~s}, 1 \mathrm{H}), 6.88(\mathrm{t},, J=6.4 \mathrm{~Hz} 1 \mathrm{H}), 6.96(\mathrm{~d},, J=8.2 \mathrm{~Hz}$ 1H), $7.20(\mathrm{~m}, 2 \mathrm{H}), 7.89(\mathrm{bs}, 1 \mathrm{H}), 8.93(\mathrm{bs}, 1 \mathrm{H})$.

${ }^{13} \mathrm{C}$ NMR (DMSO- $\left.d_{6}, 300 \mathrm{~K}, \mathrm{ppm}\right): \delta 20.6,25.9,26.0,28.8,29.2,37.4,55.6,69.5,111.0,120.2$, $127.2,128.0,128.3,157.1,168.2,171.7$.

Anal. Calcd for $\mathrm{C}_{18} \mathrm{H}_{27} \mathrm{~N}_{3} \mathrm{O}_{3}: \mathrm{C}, 64.84 ; \mathrm{H}, 8.16 ; \mathrm{N}, 12.60$. Found: $\mathrm{C}, 64.86 ; \mathrm{H}, 8.26 ; \mathrm{N}, 12.80$.

\section{2-(2-Benzoylhydrazino)-2-cyclohexyl- $N^{1}$-(2-methoxyethyl)acetamide (4m)}

Yellowish solid, mp $144-145^{\circ} \mathrm{C}$.

${ }^{1} \mathrm{H}$ NMR (DMSO-d $\left.d_{6}, 300 \mathrm{~K}, \mathrm{ppm}\right): \delta 1.07 \ldots 1.30(\mathrm{~m}, 5 \mathrm{H}), 1.56 \ldots 1.84(\mathrm{~m}, 6 \mathrm{H}), 2.97 \ldots 3.45(\mathrm{~m}, 8 \mathrm{H})$, 5.20 (bs, 1H), 7.38...7.54 (m, 3H), 7.69 (bs, 1H), 7.77 (d; , J=7.0 Hz 2H), 9.53 (bs, 1H).

${ }^{13} \mathrm{C}$ NMR (DMSO-d $\left.d_{6}, 300 \mathrm{~K}, \mathrm{ppm}\right): \delta 25.9,28.9,29.2,38.3,39.7,57.8,69.2,70.8,127.1,128.2$, 131.1, 133.6, 165.8, 171.6.

Anal. Calcd for $\mathrm{C}_{18} \mathrm{H}_{27} \mathrm{~N}_{3} \mathrm{O}_{3}: \mathrm{C}, 64.84 ; \mathrm{H}, 8.16 ; \mathrm{N}, 12.60$. Found: $\mathrm{C}, 64.84 ; \mathrm{H}, 8.10 ; \mathrm{N}, 12.46$.

Methyl 2-cyclohexyl-2-[2-(2-pyrazinylcarbonyl)hydrazino]acetate (5c). 
The compound was prepared using the same general procedure as for compounds 4a-m except methanol used as the solvent. The title compound was isolated from the reaction mixture in $13 \%$ yield by column chromatography on silica gel using $35 \rightarrow 70 \%$ ethyl acetate in hexanes as eluent.

Sticky colorless solid.

${ }^{1} \mathrm{H}$ NMR (DMSO-d $\left.6,300 \mathrm{~K}, \mathrm{ppm}\right): \delta 1.06 \ldots 1.32(\mathrm{~m}, 5 \mathrm{H}), 1.57 \ldots 1.82(\mathrm{~m}, 5 \mathrm{H}), 1.84(\mathrm{~m}, 1 \mathrm{H})$, $3.26(\mathrm{bs}, 1 \mathrm{H}), 3.51(\mathrm{~d}, J=6.2 \mathrm{~Hz}, 1 \mathrm{H}), 3.66(\mathrm{~s}, 3 \mathrm{H}), 8.66(\mathrm{~s}, 1 \mathrm{H}), 8.80(\mathrm{~s}, 1 \mathrm{H}), 9.11(\mathrm{~s}, 1 \mathrm{H}), 9.85$ (bs, 1H).

${ }^{13} \mathrm{C}$ NMR (DMSO- $\left.d_{6}, 300 \mathrm{~K}, \mathrm{ppm}\right): \delta 25.5,25.8,28.9,38.7,51.4,67.3,143.2,143.4,144.3$, $147.5,160.6,172.8$.

LC MS $\left[\mathrm{M}+\mathrm{H}^{+}\right] \mathrm{m} / \mathrm{z} 293$.

Anal. Calcd for $\mathrm{C}_{14} \mathrm{H}_{20} \mathrm{~N}_{4} \mathrm{O}_{3}$ : C,57.52; H, 6.90; N,19.16. Found: C,57.68; H, 7.04; N,19.36.

\section{Methyl 2-[2-(pyrazin-2-ylcarbonyl)hydrazino]pentanoate (5d).}

The compound was prepared using the same general procedure as for compounds $4 \mathbf{a}-\mathbf{m}$ except methanol used as the solvent. The title compound was isolated from the reaction mixture in $24 \%$ yield by column chromatography on silica gel using $35 \rightarrow 50 \%$ ethyl acetate in hexanes as eluent.

Sticky colorless solid.

${ }^{1} \mathrm{H}$ NMR (DMSO-d $\left., 300 \mathrm{~K}, \mathrm{ppm}\right): \delta 0.91(\mathrm{t}, J=7.3 \mathrm{~Hz}, 3 \mathrm{H}), 1.40(\mathrm{~m}, 2 \mathrm{H}), 1.66(\mathrm{q}, J=7.3 \mathrm{~Hz}$, 2H), 3.63 (t, $J=7.1 \mathrm{~Hz}, 1 \mathrm{H}), 3.66$ (s, 3H), 4.95 (bs, 1H), 7.70 (d, $J=4.9 \mathrm{~Hz} 2 \mathrm{H}), 8.70$ (d, $J=4.9$ $\mathrm{Hz} 2 \mathrm{H}), 10.00$ (bs, 1H).

${ }^{13} \mathrm{C}$ NMR (DMSO- $d_{6}, 300 \mathrm{~K}$ ppm): $\delta 13.6,18.3,32.3,51.6,62.0,121.6,141.1,149.0,163.3$, 172.8 .

LC MS $\left[\mathrm{M}+\mathrm{H}^{+}\right] \mathrm{m} / \mathrm{z} 252$.

Anal. Calcd for $\mathrm{C}_{12} \mathrm{H}_{17} \mathrm{~N}_{3} \mathrm{O}_{3}$ : C,57.36; H,6.82; N,16.72. Found: C,57.50; H,6.94; N,16.67.

\section{4-[2-(Cyclohexylmethylene)hydrazino]-4-oxobutanoic acid (6)}

tert-Butyl carbazate $(1,320 \mathrm{mg}, 10 \mathrm{mmol})$ was dissolved in DCM $(50 \mathrm{~mL})$ and the solution was cooled to $5^{\circ} \mathrm{C}$. Ethyl 4-chloro-4-oxobutyrate $(2.15 \mathrm{~g}, 13 \mathrm{mmol})$ was added dropwise so as to maintain the temperature of the reaction mixture below $10^{\circ} \mathrm{C}$. The reaction mixture was warmed up to r. t. and stirred for $1 \mathrm{~h}$. The reaction mixture was washed with $10 \%$ aq. $\mathrm{Na}_{2} \mathrm{CO}_{3}(100 \mathrm{ml})$, water $(100 \mathrm{ml})$, dried over anhydrous $\mathrm{MgSO}_{4}$, and concentrated in vacuo. The residue was dissolved in dioxane $(10 \mathrm{~mL})$ and treated with $4 \mathrm{M} \mathrm{HCl}$ in dioxane $(10 \mathrm{ml})$. The reaction mixture was stirred at r. t. overnight, the resulting precipitate was collected by filtration and dissolved in methanol $(20 \mathrm{~mL})$. Cyclohexanecarboxaldexyde $(1.2 \mathrm{~mL}, 10 \mathrm{mmol})$ and triethylamine $(1.44 \mathrm{~mL}$, $10 \mathrm{mmol}$ ) were added and the resulting mixture was stirred at $\mathrm{r}$. t. for 2 hours. The solvent was 
removed in vacuo and the residue was dispersed in water, filtered off and the filter cake was washed with hexane. The solid product was dissolved in methanol $(10 \mathrm{~mL})$ and the solution of $\mathrm{NaOH}(400 \mathrm{mg}, 10 \mathrm{mmol})$ in water $(5 \mathrm{ml})$ was added. The resulting mixture was stirred at $\mathrm{r} . \mathrm{t}$. for $3 \mathrm{~h}$, the solvent was removed under reduced pressure, and the residue was re-dissolved in water $(30 \mathrm{~mL})$, filtered, and the filtrate was carefully neutralized with $5 \%$ aq. $\mathrm{HCl}$. The resulting precipitate was filtered off. Additional crystallization from methanol provided 6 (720 mg, 32\% over 4 steps).

Amber solid, mp $147-149{ }^{\circ} \mathrm{C}$.

${ }^{1} \mathrm{H}$ NMR (DMSO-d $\left.6,363 \mathrm{~K}, \mathrm{ppm}\right): \delta 1.19 \ldots 1.42(\mathrm{~m}, 5 \mathrm{H}), 1.59 \ldots 1.85(\mathrm{~m}, 5 \mathrm{H}), 2.21(\mathrm{~m}, 1 \mathrm{H})$, 2.41...2.76 (m, 4H), 5.17 (bs, 2H), 7.31 (bs, 1H), 10.28 (bs, 1H).

${ }^{13} \mathrm{C}$ NMR (DMSO-d 6 , $\left.363 \mathrm{~K}, \mathrm{ppm}\right): \delta 24.8,25.5,28.3,28.9,39.7,151.3,172.1,173.4$.

\section{$N$-(tert-Butyl)-2-cyclohexyl-2-(3,6-dioxotetrahydropyridazin-1(2H)-yl)acetamide (7)}

6 (340 mg, $1.5 \mathrm{mmol})$ was dissolved in isopropanol $(5 \mathrm{~mL})$ and tert-butyl isocyanide $(200 \mathrm{~mL}$, $2.1 \mathrm{mmol}$ ) was added. The reaction mixture was stirred at $50{ }^{\circ} \mathrm{C}$ overnight. The solvent was removed under reduced pressure and the product was purified by column chromatography on silica gel using $0 \rightarrow 2.5 \%$ methanol in chloroform as eluent to provide 7 (334 $\mathrm{mg}, 72 \%$ ).

White solid, mp $129-131{ }^{\circ} \mathrm{C}$.

${ }^{1} \mathrm{H}$ NMR (DMSO- $\left.d_{6}, 300 \mathrm{~K}, \mathrm{ppm}\right): \delta 1.08 \ldots 1.32(\mathrm{~m}, 14 \mathrm{H}), 1.59 \ldots 1.79(\mathrm{~m}, 6 \mathrm{H}), 2.53 \ldots 2.66(\mathrm{~m}$, $4 \mathrm{H}), 5.46(\mathrm{~d}, J=1.5 \mathrm{~Hz}, 1 \mathrm{H}), 7.58(\mathrm{~s}, 1 \mathrm{H})$.

${ }^{13} \mathrm{C}$ NMR (DMSO-d 6 , $\left.300 \mathrm{~K}, \mathrm{ppm}\right): \delta 25.8,26.1,28.2,28.4,28.9,39.6,49.8,67.1,95.5,164.2$, $170.0,175.3$.

LC MS $\left[\mathrm{M}+\mathrm{H}^{+}\right] \mathrm{m} / \mathrm{z} 310$.

Anal. Calcd for $\mathrm{C}_{16} \mathrm{H}_{27} \mathrm{~N}_{3} \mathrm{O}_{3}$ : C, 56.44; H, 8.35; N, 11.52. Found: C, 56.11; H, 8.27; N, 11.48.

\section{2-Cyclohexyl-2-[2-isonicotinoyl-1-(3-methylbutyl)hydrazino]- $N$-(2-methoxyethyl)acetamide (9a); Typical Procedure for the Synthesis of Compounds 9a-k.}

4a (335 mg, $1 \mathrm{mmol})$ was dissolved in DCM (5 mL). Isovaleric aldehyde (165 mL, $1.5 \mathrm{mmol})$ was added followed by $\mathrm{NaBH}(\mathrm{OAc})_{3}(380 \mathrm{mg}, 2 \mathrm{mmol})$. The resulting mixture was stirred at $\mathrm{r}$. $\mathrm{t}$. overnight, diluted with DCM $(25 \mathrm{~mL})$, washed with water $(3 \times 25 \mathrm{~mL})$, dried over anydrous $\mathrm{MgSO}_{4}$, filtered and concentrated in vacuo to provide crude product. The latter was purified by column chromtography on silica unsing $0 \rightarrow 5 \%$ methanol in chloroform as eluent to provide 9a (250 mg, 62\%).

White solid, mp $99-101{ }^{\circ} \mathrm{C}$. 
${ }^{1} \mathrm{H}$ NMR (DMSO- $\left.d_{6}, 363 \mathrm{~K}, \mathrm{ppm}\right): \delta 0.88(\mathrm{~d}, J=6.6 \mathrm{~Hz}, 6 \mathrm{H}), 0.96 \ldots 1.20(\mathrm{~m}, 5 \mathrm{H}), 1.38(\mathrm{~m}, 2 \mathrm{H})$, $1.46 \ldots 1.82(\mathrm{~m}, 6 \mathrm{H}), 2.20(\mathrm{~m}, 1 \mathrm{H}), 2.73(\mathrm{~m}, 2 \mathrm{H}), 3.18(\mathrm{~d}, J=9.5 \mathrm{~Hz} 1 \mathrm{H}), 3.27(\mathrm{~s}, 1 \mathrm{H}), 3.33(\mathrm{t}, J=$ $5.2 \mathrm{~Hz}, 2 \mathrm{H}), 3.41(\mathrm{t}, J=5.2 \mathrm{~Hz}, 2 \mathrm{H}), 7.60(\mathrm{~d}, J=5.4 \mathrm{~Hz}, 2 \mathrm{H}), 8.1(\mathrm{bs}, 1 \mathrm{H}), 8.71(\mathrm{~d}, J=5.4 \mathrm{~Hz}$, 2H), $9.4(\mathrm{bs}, 1 \mathrm{H})$.

${ }^{13} \mathrm{C}$ NMR (DMSO-d $\left.6,363 \mathrm{~K}, \mathrm{ppm}\right): \delta 22.3,25.4,26.1,29.0,29.9,35.6,37.2,38.1,54.3,57.8$, $70.6,70.7,120.6,141.4,150.3,162.8,172.3$.

Anal. Calcd for $\mathrm{C}_{22} \mathrm{H}_{36} \mathrm{~N}_{4} \mathrm{O}_{3}$ : C, 65.32; H, 8.97; N, 13.85. Found: C, 65.40; H, 9.04; N, 13.90.

\section{2-Cyclohexyl-2-1-isopentyl-2-[2-(4-methylphenyl)acetyl]hydrazino- $N^{1}$-(2- methoxyethyl)acetamide (9b)}

White solid, mp $126-128{ }^{\circ} \mathrm{C}$.

${ }^{1} \mathrm{H}$ NMR (DMSO- $\left.d_{6}, 363 \mathrm{~K}, \mathrm{ppm}\right): \delta 0.68 \ldots 0.91(\mathrm{~m}, 8 \mathrm{H}), 0.93 \ldots 1.69(\mathrm{~m}, 11 \mathrm{H}), 2.04(\mathrm{~m}, 1 \mathrm{H})$, $2.25(\mathrm{~s}, 3 \mathrm{H}), 2.56(\mathrm{~m}, 2 \mathrm{H}), 2.94(\mathrm{~d}, J=9.5 \mathrm{~Hz}, 1 \mathrm{H}), 3.17 \ldots 3.41(\mathrm{~m}, 9 \mathrm{H}), 7.08(\mathrm{~d}, J=8.21 \mathrm{~Hz}$, 2H), 7.14 (d, $J=8.21 \mathrm{~Hz}, 2 \mathrm{H}), 8.18$ (bs, 1H) 8.50 (s, 1H).

${ }^{13} \mathrm{C}$ NMR (DMSO- $\left.d_{6}, 363 \mathrm{~K}, \mathrm{ppm}\right): \delta 20.6,22.4,25.2,26.2,28.8,29.8,35.5,36.6,37.8,41.1$, $54.1,57.8,70.5,71.0,128.7,128.8,133.1,135.4,168.5,172.1$.

Anal. Calcd for $\mathrm{C}_{25} \mathrm{H}_{41} \mathrm{~N}_{3} \mathrm{O}_{2}$ : C, 69.57; H, 9.57; N, 9.74. Found: C, 69.66; H, 9.61; N, 9.88.

\section{2-[1-Butyl-2-(pyrazin-2-ylcarbonyl)hydrazino]-2-cyclohexyl- $N$-(2-methoxyethyl)acetamide} (9c)

Grey solid, mp $137-139^{\circ} \mathrm{C}$.

${ }^{1} \mathrm{H}$ NMR (DMSO-d $\left.d_{6}, 363 \mathrm{~K}, \mathrm{ppm}\right): \delta 0.88(\mathrm{t}, J=6.6 \mathrm{~Hz}, 3 \mathrm{H}), 0.94 \ldots 1.18(\mathrm{~m}, 5 \mathrm{H}), 1.33 \ldots 1.71$ $(\mathrm{m}, 9 \mathrm{H}), 2.25(\mathrm{~m}, 1 \mathrm{H}), 2.73(\mathrm{~m}, 2 \mathrm{H}), 3.18(\mathrm{~d}, J=9.5 \mathrm{~Hz} 1 \mathrm{H}), 3.27(\mathrm{~s}, 3 \mathrm{H}), 3.33(\mathrm{t}, J=5.2 \mathrm{~Hz}$, 2H), $3.41(\mathrm{t}, J=5.2 \mathrm{~Hz}, 2 \mathrm{H}), 8.05(\mathrm{bsH}), 8.68(\mathrm{~s}, 1 \mathrm{H}), 8.82(\mathrm{~s}, 1 \mathrm{H}), 9.17(\mathrm{~s}, 1 \mathrm{H}), 10.14(\mathrm{bs}, 1 \mathrm{H})$.

${ }^{13} \mathrm{C}$ NMR (DMSO-d 6 , $\left.363 \mathrm{~K}, \mathrm{ppm}\right): \delta 13.7,19.6,25.2,26.1,28.8,29.6,36.7,37.9,56.2,57.8$, 69.7 70.6, 143.3, 143.4, 144.4, 147.7, 160.0, 172.6.

Anal. Calcd for $\mathrm{C}_{20} \mathrm{H}_{33} \mathrm{~N}_{5} \mathrm{O}_{3}$ : C, 61.36; H, 8.50; N, 17.89. Found: C, 61.43; H, 8.60; N, 17.95.

\section{2-[2-Isonicotinoyl-1-(3-methylbutyl)hydrazino]- $N$-(2-methoxyethyl)pentanamide (9d)}

Beige solid, mp $148^{\circ} \mathrm{C}$ (decomp.).

${ }^{1} \mathrm{H}$ NMR (DMSO- $\left.d_{6}, 363 \mathrm{~K}, \mathrm{ppm}\right): \delta 0.86(\mathrm{~m}, 9 \mathrm{H}), 1.25 \ldots 1.47(\mathrm{~m}, 4 \mathrm{H}), 1.50 \ldots 1.79(\mathrm{~m}, 3 \mathrm{H}), 2.81$ $(\mathrm{m}, 2 \mathrm{H}), 3.26(\mathrm{~s}, 3 \mathrm{H}), 3.29(\mathrm{~m}, 2 \mathrm{H}), 3.35 \ldots 3.47(\mathrm{~m}, 3 \mathrm{H}), 7.62(\mathrm{~d}, J=3.7 \mathrm{~Hz}, 2 \mathrm{H}), 8.0(\mathrm{bs}, 1 \mathrm{H})$, $8.70(\mathrm{~d}, J=3.7 \mathrm{~Hz}, 2 \mathrm{H}), 9.3(\mathrm{bs}, 1 \mathrm{H})$. 
${ }^{13} \mathrm{C}$ NMR (DMSO- $\left.d_{6}, 363 \mathrm{~K}, \mathrm{ppm}\right): \delta 13.7,18.8,22.3,25.3,31.7,36.0,38.2,39.2,53.3,57.7$, $66.8,70.6,120.9,141.2,150.2,163.9,172.8$.

Anal. Calcd for $\mathrm{C}_{19} \mathrm{H}_{32} \mathrm{~N}_{4} \mathrm{O}_{3}$ : C, 62.61; H, 8.85; N, 15.37. Found: C, 62.55; H, 8.92; N, 15.38.

\section{2-(1-Butyl-2-isonicotinoylhydrazino)- $N$-(2-methoxyethyl)pentanamide (9e)}

Yellow solid, mp $128-130{ }^{\circ} \mathrm{C}$.

${ }^{1} \mathrm{H}$ NMR (DMSO- $\left.d_{6}, 363 \mathrm{~K}, \mathrm{ppm}\right): \delta 0.86(\mathrm{~m}, 6 \mathrm{H}), 1.30 \ldots 1.50(\mathrm{~m}, 6 \mathrm{H}), 1.60(\mathrm{~m}, 2 \mathrm{H}), 2.80(\mathrm{~m}$, $2 \mathrm{H}), 3.21 \ldots 3.24(\mathrm{~m}, 5 \mathrm{H}), 3.35 \ldots 3.47(\mathrm{~m}, 3 \mathrm{H}), 7.63(\mathrm{~d}, J=3.8 \mathrm{~Hz}, 2 \mathrm{H}), 7.97(\mathrm{bs}, 1 \mathrm{H}), 8.71(\mathrm{~d}, J=$ $3.8 \mathrm{~Hz}, 2 \mathrm{H}), 9.3(\mathrm{bs}, 1 \mathrm{H})$.

${ }^{13} \mathrm{C}$ NMR (DMSO- $\left.d_{6}, 363 \mathrm{~K}, \mathrm{ppm}\right): \delta 13.5,13.6,18.8,19.6,29.1,31.7,38.2,54.7,57.8,66.9$, $70.6,120.9,141.2,150.1,163.9,172.8$.

Anal. Calcd for $\mathrm{C}_{18} \mathrm{H}_{30} \mathrm{~N}_{4} \mathrm{O}_{3}: \mathrm{C}, 61.69 ; \mathrm{H}, 8.63 ; \mathrm{N}, 15.99$. Found: $\mathrm{C}, 61.56 ; \mathrm{H}, 8.71 ; \mathrm{N}, 16.07$.

\section{N-Benzyl-2-cyclohexyl-2-[1-(3-methylbutyl)-2-(pyrazin-2-ylcarbonyl)hydrazino]acetamide} (9f)

Coloress foam, mp $118-120{ }^{\circ} \mathrm{C}$.

${ }^{1} \mathrm{H}$ NMR (DMSO-d $\left.6,363 \mathrm{~K}, \mathrm{ppm}\right): \delta 0.86\left(\mathrm{dd}, J_{1}=6.9 \mathrm{~Hz}, J_{2}=2.7 \mathrm{~Hz} 6 \mathrm{H}\right), 0.94 \ldots 1.19(\mathrm{~m}, 5 \mathrm{H})$, $1.27 \ldots 1.78(\mathrm{~m}, 8 \mathrm{H}), 2.24(\mathrm{~m}, 1 \mathrm{H}), 2.78(\mathrm{~m}, 2 \mathrm{H}), 3.24(\mathrm{~d}, J=9.9 \mathrm{~Hz}, 1 \mathrm{H}), 4.37$ (ddd, $J_{1}=19.2$ $\left.\mathrm{Hz}, J_{2}=5.6 \mathrm{~Hz}, J_{3}=5.6 \mathrm{~Hz}, 2 \mathrm{H}\right), 7.20 \ldots 7.35(\mathrm{~m}, 5 \mathrm{H}), 8.58(\mathrm{bs}, 1 \mathrm{H}), 8.68(\mathrm{~s}, 1 \mathrm{H}) 8.83(\mathrm{~d}, J=$ $1.9 \mathrm{~Hz}, 1 \mathrm{H}), 9.17(\mathrm{~s}, 1 \mathrm{H}), 10.18(\mathrm{~s}, 1 \mathrm{H})$.

${ }^{13} \mathrm{C}$ NMR (DMSO-d $\left., 363 \mathrm{~K}, \mathrm{ppm}\right): \delta 22.4,25.3,26.1,29.2,29.6,35.6,36.8,41.8,54.9,69.6$, $126.9,127.4,128.2,139.0,143.4,143.4,144.4,147.7,160.0,172.5$.

Anal. Calcd for $\mathrm{C}_{25} \mathrm{H}_{35} \mathrm{~N}_{5} \mathrm{O}_{2}$ : C, 68.62; H, 8.06; N, 16.00. Found: C, 68.70; H, 8.18; N, 15.77.

\section{N-Benzyl-2-[1-butyl-2-(pyrazin-2-ylcarbonyl)hydrazino]-2-cyclohexylacetamide (9g)}

Grey solid, mp $129-131{ }^{\circ} \mathrm{C}$.

${ }^{1} \mathrm{H}$ NMR (DMSO-d $\left.6,363 \mathrm{~K}, \mathrm{ppm}\right): \delta 0.86(\mathrm{t}, J=7.0 \mathrm{~Hz}, 3 \mathrm{H}), 0.93 \ldots 1.20(\mathrm{~m}, 5 \mathrm{H}), 1.28 \ldots 1.72(\mathrm{~m}$, 9H), $2.24(\mathrm{~m}, 1 \mathrm{H}), 2.75(\mathrm{~m}, 2 \mathrm{H}), 3.23(\mathrm{~d}, J=9.1 \mathrm{~Hz}, 1 \mathrm{H}), 4.4\left(\mathrm{ddd}, J_{1}=9.6 \mathrm{~Hz}, J_{2}=4.8 \mathrm{~Hz}, J_{3}=\right.$ $4.8 \mathrm{~Hz}, 2 \mathrm{H}), 7.18 \ldots 7.37(\mathrm{~m}, 5 \mathrm{H}), 8.54(\mathrm{bs}, 1 \mathrm{H}), 8.68(\mathrm{~s}, 1 \mathrm{H}) 8.83(\mathrm{~s}, 1 \mathrm{H}), 9.16(\mathrm{~s}, 1 \mathrm{H}), 10.15(\mathrm{~s}$, $1 \mathrm{H})$.

${ }^{13} \mathrm{C}$ NMR (DMSO-d 6 , $\left.363 \mathrm{~K}, \mathrm{ppm}\right): \delta 13.7,19.6,25.2,26.0,28.8,36.8,41.8,56.3,69.7,126.9$, $127.4,128.3,139.0,143.4,144.4,147.7,160.0,172.5$.

Anal. Calcd for $\mathrm{C}_{24} \mathrm{H}_{33} \mathrm{~N}_{5} \mathrm{O}_{2}$ : C, 68.06; H, 7.85; N, 16.53. Found: C, 68.01; H, 7.73; N, 16.64. 


\section{$N$-Benzyl-2-cyclohexyl-2-[2-isonicotinoyl-1-(3-methylbutyl)hydrazino]acetamide (9h)}

White solid, mp $146{ }^{\circ} \mathrm{C}$ (decomp.).

${ }^{1} \mathrm{H}$ NMR (DMSO-d $\left.d_{6}, 363 \mathrm{~K}, \mathrm{ppm}\right): \delta 0.86(\mathrm{~d}, J=6.4 \mathrm{~Hz}, 6 \mathrm{H}), 0.96 \ldots 1.19(\mathrm{~m}, 5 \mathrm{H}), 1.38(\mathrm{~m}, 2 \mathrm{H})$, $1.47 \ldots 1.78(\mathrm{~m}, 6 \mathrm{H}), 2.20(\mathrm{~m}, 1 \mathrm{H}), 2.80\left(\mathrm{q}, J_{1}=7.1 \mathrm{~Hz}, J_{2}=7.3 \mathrm{~Hz}, 2 \mathrm{H}\right), 3.24(\mathrm{~d}, J=9.6 \mathrm{~Hz}, 1 \mathrm{H})$, $4.4\left(\mathrm{ddd}, J_{1}=11.9 \mathrm{~Hz}, J_{2}=6.0 \mathrm{~Hz}, J_{3}=5.8 \mathrm{~Hz} 2 \mathrm{H}\right), 7.20 \ldots 7.34(\mathrm{~m}, 5 \mathrm{H}), 7.61(\mathrm{~d}, J=4.8 \mathrm{~Hz}, 2 \mathrm{H})$, 8.53 (bs, 1H), 8.72 (d, $J=4.8 \mathrm{~Hz}, 2 \mathrm{H}), 9.4$ (bs, $1 \mathrm{H})$.

${ }^{13} \mathrm{C}$ NMR (DMSO-d $\left., 363 \mathrm{~K}, \mathrm{ppm}\right): \delta 22.4,25.2,26.1,29.1,29.8,35.7,37.1,44.9,54.1,70.4$, $121.1,126.9,127.4,128.2,138.9,141.6,149.9,162.5,172.2$.

Anal. Calcd for $\mathrm{C}_{26} \mathrm{H}_{36} \mathrm{~N}_{4} \mathrm{O}_{2}:$ C, 71.53; H, 8.31; N, 12.83. Found: C, 71.55; H, 8.36; N, 12.97 .

\section{$N$-Benzyl-2-(1-butyl-2-isonicotinoylhydrazino)-2-cyclohexylacetamide (9i)}

White solid, mp $157{ }^{\circ} \mathrm{C}$ (decomp.).

${ }^{1} \mathrm{H}$ NMR (DMSO- $\left.d_{6}, 363 \mathrm{~K}, \mathrm{ppm}\right): \delta 0.87$ (t, $\left.J=7.0 \mathrm{~Hz}, 3 \mathrm{H}\right), 0.96 \ldots 1.22(\mathrm{~m}, 5 \mathrm{H}), 1.34 \ldots 1.76(\mathrm{~m}$, $9 \mathrm{H}), 2.20(\mathrm{~m}, 1 \mathrm{H}), 2.80\left(\mathrm{q}, J_{1}=6.4 \mathrm{~Hz}, J_{2}=5.9 \mathrm{~Hz}, 2 \mathrm{H}\right), 3.24(\mathrm{~d}, J=9.2 \mathrm{~Hz}, 1 \mathrm{H}), 4.4$ (ddd, $J_{1}=$ $\left.7.0 \mathrm{~Hz}, J_{2}=5.5 \mathrm{~Hz}, J_{3}=5.5 \mathrm{~Hz} 2 \mathrm{H}\right), 7.20 \ldots 7.34(\mathrm{~m}, 5 \mathrm{H}), 7.63(\mathrm{~d}, J=7.3 \mathrm{~Hz}, 2 \mathrm{H}), 8.50(\mathrm{bs}, 1 \mathrm{H})$, $8.73(\mathrm{~d}, J=4.8 \mathrm{~Hz}, 2 \mathrm{H}), 9.4(\mathrm{bs}, 1 \mathrm{H})$.

${ }^{13} \mathrm{C}$ NMR (DMSO-d $\left., 363 \mathrm{~K}, \mathrm{ppm}\right): \delta 13.5,19.5,25.3,26.0,29.0,29.9,37.4,42.2,55.7,70.8$, $120.8,126.8,127.5,128.1,138.9,141.8,149.9,162.8,172.2$.

Anal. Calcd for $\mathrm{C}_{25} \mathrm{H}_{34} \mathrm{~N}_{4} \mathrm{O}_{2}$ : C, 71.06; H, 8.11; N, 13.26. Found: C, 70.99; H, 8.03; N, 13.34.

\section{N-Benzyl-2-[2-isonicotinoyl-1-(3-methylbutyl)hydrazino]pentanamide (9j)}

Beige solid, mp $133-134{ }^{\circ} \mathrm{C}$.

${ }^{1} \mathrm{H}$ NMR (DMSO-d $\left.d_{6}, 363 \mathrm{~K}, \mathrm{ppm}\right): \delta 0.81 \ldots 0.91(\mathrm{~m}, 9 \mathrm{H}), 1.29 \ldots 1.49(\mathrm{~m}, 4 \mathrm{H}), 1.54 \ldots 1.74(\mathrm{~m}$, $3 \mathrm{H}), 2.85(\mathrm{~m}, 2 \mathrm{H}), 3.51(\mathrm{t}, J=6.0 \mathrm{~Hz}, 1 \mathrm{H}), 4.34\left(\mathrm{ddd}, J_{1}=5.5 \mathrm{~Hz}, J_{2}=4.1 \mathrm{~Hz}, J_{3}=4.8 \mathrm{~Hz} 2 \mathrm{H}\right)$, $7.19 \ldots 7.23(\mathrm{~m}, 5 \mathrm{H}), 7.64(\mathrm{~d}, J=5.8 \mathrm{~Hz}, 2 \mathrm{H}), 8.45(\mathrm{bs}, 1 \mathrm{H}), 8.72(\mathrm{~d}, J=5.8 \mathrm{~Hz}, 2 \mathrm{H}), 9.34$ (bs, $1 \mathrm{H})$.

${ }^{13} \mathrm{C}$ NMR (DMSO-d 6 , $\left.363 \mathrm{~K}, \mathrm{ppm}\right): \delta 13.6,18.8,22.3,25.3,31.7,36.0,42.2,53.4,66.8,121.1$, $126.7,127.3,128.1,139.1,141.5,149.8,163.8,172.8$.

Anal. Calcd for $\mathrm{C}_{23} \mathrm{H}_{32} \mathrm{~N}_{4} \mathrm{O}_{2}$ : C, 69.67; H, 8.13; N, 14.13. Found: C, 69.55; H, 8.07; N, 14.19.

\section{$N$-Benzyl-2-(1-butyl-2-isonicotinoylhydrazino)pentanamide (9k)}

Yellowish solid, $\mathrm{mp} 150{ }^{\circ} \mathrm{C}$ (decomp.). 
${ }^{1} \mathrm{H}$ NMR (DMSO- $\left.d_{6}, 363 \mathrm{~K}, \mathrm{ppm}\right): \delta 0.87(\mathrm{~m}, 6 \mathrm{H}), 1.28 \ldots 1.51(\mathrm{~m}, 6 \mathrm{H}), 1.45(\mathrm{~m}, 2 \mathrm{H}), 2.82(\mathrm{~m}$, $2 \mathrm{H}), 3.50(\mathrm{t}, J=6.3 \mathrm{~Hz}, 1 \mathrm{H}), 4.35(\mathrm{~d}, J=5.8 \mathrm{~Hz}, 2 \mathrm{H}), 7.17 \ldots 7.33(\mathrm{~m}, 5 \mathrm{H}), 7.63(\mathrm{~d}, J=4.4 \mathrm{~Hz}$, 2H), $8.43(\mathrm{bs}, 1 \mathrm{H}), 8.70(\mathrm{~d}, J=4.4 \mathrm{~Hz}, 2 \mathrm{H}), 9.34(\mathrm{bs}, 1 \mathrm{H})$.

${ }^{13} \mathrm{C}$ NMR (DMSO-d 6 , $\left.363 \mathrm{~K}, \mathrm{ppm}\right): \delta 13.5,13.6,18.8,19.6,29.1,31.7,42.2,54.8,66.8,121.0$, $126.7,127.3,128.1,139.1,141.4,150.0,164.0,172.3$.

Anal. Calcd for $\mathrm{C}_{22} \mathrm{H}_{30} \mathrm{~N}_{4} \mathrm{O}_{2}$ : C, 69.08; H, 7.91; N, 14.65. Found: C, 68.93; H, 8.00; $\mathrm{N}, 14.52$.

\section{Acknowledgment}

This research was supported by the Federal Agency for Science and Innovation (Russian Federation Government Contract 02.740.11.0092). Dr. Alexander Manaev of Chemical Diversity Research Institute is acknowledged for his help in obtaining X-ray crystallography data. Dr. Yan Ivanenkov of Chemical Diversity research institute is thanked for performing molecular mechanics calculations.

\section{References and notes.}

1. Ugi, I.; Meyr, R.; Fitzer, U.; Steinbrucker, C. Angew. Chem. 1959, 71, 386-390.

2. Dömling, A. Chem. Rev. 2006, 106, 17-89.

3. El Kaim, L.; Grimaud, L. Tetrahedron 2009, 65, 2153-2171.

4. (a) Ugi, I.; Bodesheim, F. Chem. Ber. 1961, 94, 2797-2718. (b) Ugi, I.; Bodesheim, F. Justus Liebigs Ann. Chem. 1963, 666, 61-64. (c) Zinner, G.; Kliegel, W. Arch. Pharm. 1966, 299, 746-756. (d) Zinner, G; Bock, W. Arch. Pharm. 1971, 304, 933-943. (e) Failli, A.; Nelson, V.; Immer, H.; Götz, M. Can. J. Chem. 1973, 51, 2769-2775. (f) Marcaccini, S.; Pepino, R.; Polo, C.; Pozo, M. C. Synthesis 2001, 85-88.

5. a) Zinner, G.; Moderhack, D.; Kliegel, W. Chem. Ber. 1969, 102, 2536-2546; (b) Moderhack, D. Liebigs Ann. Chem. 1973, 764, 359-364; (c) Zinner, G.; Moderhack, D.; Hantelmann, O.; Bock, W. Chem. Ber. 1974, 107, 2947-2955. (d) Basso, A.; Banfi, L.; Guanti, G.; Riva, R.; Riu, A. Tetrahedron Lett. 2004, 45, 6109-6111. (e) Basso, A.; Banfi, L.; Guanti, G.; Riva, R. Tetrahedron Lett. 2005, 46, 8003-8006.

6. Diaz, J. L.; Miguel, M.; Lavilla, R. J. Org. Chem. 2004, 69, 3550-3553.

7. Kiselyov, A. S. Tetrahedron Lett. 2005, 46, 4851-4854.

8. Grupe, R.; Baeck, B.; Niedrich, H. J. Prakt. Chem. 1971, 314, 751-758. 
9. Bushkova, E.; Parchinsky, V.; Krasavin, M. Mol. Diversity, in press.

10. Lelais, G.; Seebach, D. Helv. Chim. Acta 2003, 86, 4152-4168.

11. Guy, L.; Vidal, J.; Collet, A. J. Med. Chem. 1998, 41, 4833-4843.

12. Aubury, A.; Mangeot, J.-P.; Vidal, J.; Collet, A.; Zerkout, S.; Marraud, M. Int. J. Pepetide \& Protein Res. 1994, 43, 305-311.

13. Efforts are currently underway in our laboratories to realize a strategy of stereochemistry relay from non-racemic partners in the hydrazine-Ugi reaction and thus prepare short nonracemic hydrazinopeptide compounds via diastereomeric resolution.

14. Dömling, A.; Ugi, I. Angew. Chem. Int. Ed. 2000, 39, 3168-3210.

15. (a) Marcaccini,S. ; Miguel, D. ; Torroba, T. ; Garcia-Valverde, M. J. Org.Chem. 2003, 68, 3315-3318. (b) Marcaccini, S.; Pepino, R.; Torroba, T.; Miguel, D.; Garcia-Valverde, M. Tetrahedron Lett. 2002, 43, 8591-8593. (c) Ilyin, A. P.; Trifilenkov, A.; Kurashvili, I.; Krasavin M.; Ivachtchenko, A. V. J. Comb. Chem. 2005, 7, 360-363. (d) Ilyin, A. P.; Loseva, M. V.; Vvedensky, V. Y.; Putsykina, E. B.; Tkachenko, S. E.; Kravchenko, D. V.; Khvat, A. V.; Krasavin M.; Ivachtchenko, A. V. J. Org. Chem. 2006, 71, 2811-2819.

16. Naskar, D.; Roy, A.; Seibel, W. L.; West, L.; Portlock. D. E. Tetrahedron Lett. 2003, 44, 6297-6300.

17. (a) Novak, P.; Piculjan, K.; Hrenar, T.; Biljan, T.; Meic, Z. J. Mol. Structure 2009, 919, 6671. (b) Abraham, R. J.; Mobli, M. Magn. Res. Chem. 2007, 45, 865-877. (c) Grzesiek, S.; Cordier, F.; Jaravine, V.; Barfield, M. Prog. Nucl. Magn. Res. Spectroscopy 2004, 45, $275-$ 300. (d) Samoilenko, A. A. Zhurnal Strukturnoi Khimii 1975, 16, 568-571 (in Russian).

18. ${ }^{1} \mathrm{H}$ NMR chemical shifts Less acidic $\mathrm{N}(\alpha)$-H proton in $\mathbf{4}$ as well as the analogous $\mathrm{N}-\mathrm{H}$ proton in $\mathbf{1 0}$ were found negligibly sensitive to the solvent change, most likely due to their being less acidic.

19. All reference fragments $\mathbf{1 0}$ and $\mathbf{1 1}$ used in this work are known and commercially available compounds.

20. Crystallographic data (excluding structure factors) for the structure $\mathbf{4 i}$ and $\mathbf{9 h}$ in this paper have been deposited with the Cambridge Crystallographic Data Centre as supplementary publications CCDC 743067 and CCDC 752329, respectively. Copies of the data can be 
obtained, free of charge, on application to CCDC, 12 Union Road, Cambridge CB2 1EZ, UK, (fax: +44-(0)1223-336033 or e-mail: deposit@,ccdc.cam.ac.uk).

21. Molecular mechanics (MM2) calculations performed using ChemBio3D (Ultra) v. 11.0 demonstrated that for $\mathbf{4 i}$ and $\mathbf{9 h}$, the observed conformations displayed minimized energies of 6.48 and $7.62 \mathrm{kcal} / \mathrm{mol}$, respectively. Alternative hydrogen-bonded conformations $4 \mathbf{i}$ ' and 9h' displayed significantly higher minimized energies (14.1 and $14.0 \mathrm{kcal} / \mathrm{mol}$, respectively):
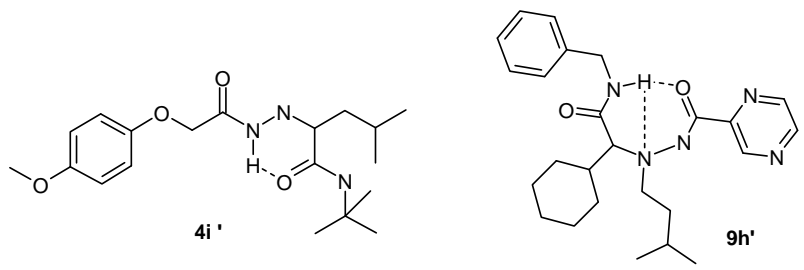

Figure 1. Hydrazinopeptides and their 'hydrazino turn' conformation.
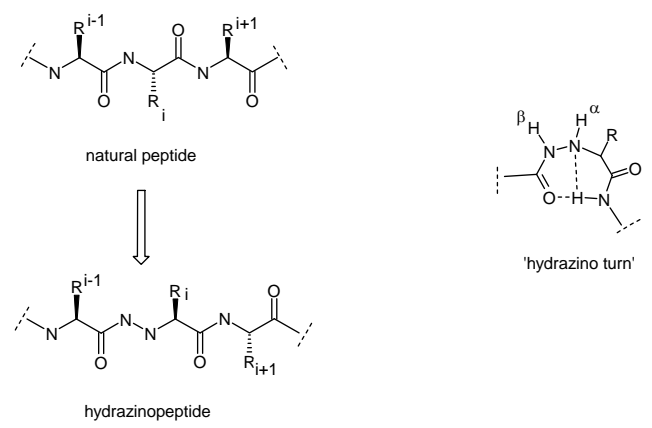

Figure 3. Markedly different secondary structure patterns observed for compounds $\mathbf{4 i}$ and $\mathbf{9 h}$ as confirmed by X-ray crystallographic analysis.
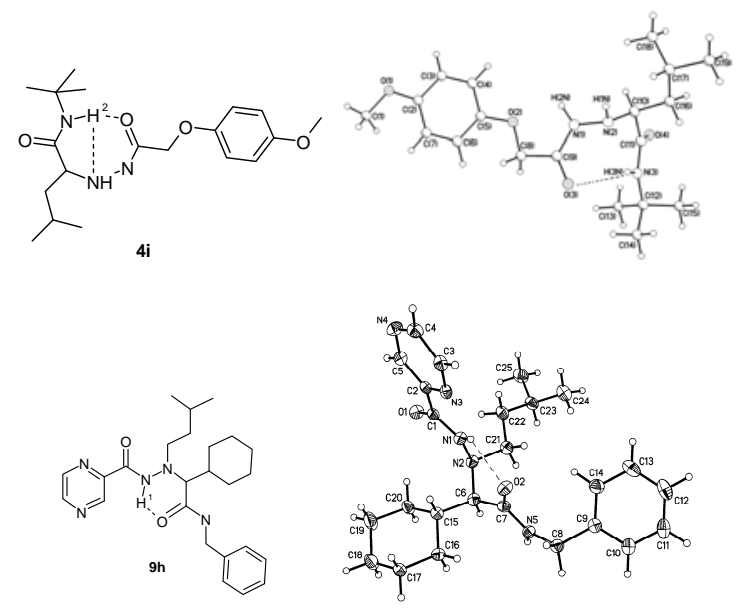
Figure 2. Structures of $\mathbf{4}$ and $\mathbf{9}$ units and the respective reference fragments for the ${ }^{1} \mathrm{H}$ NMR study.
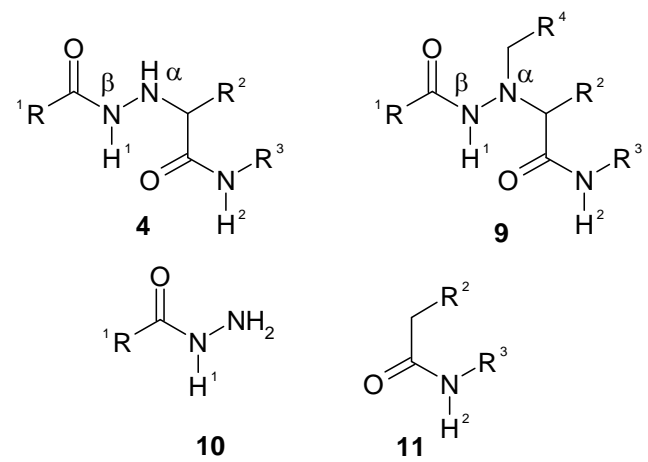

Scheme 1. Synthesis of $N^{(\alpha)}$-alkyl, $N^{(\beta)}$-acyl hydrazines 4 as starting materials for the second Ugi reaction. ${ }^{9}$

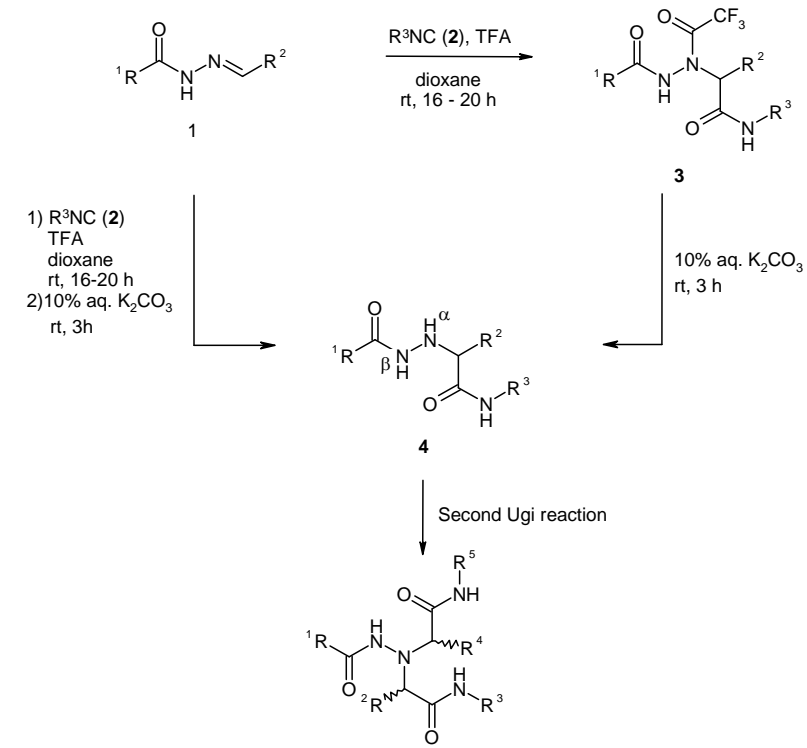

Scheme 2. Hydrazino-Ugi reaction in methanol.

${ }_{{ }_{1}^{2}} \overbrace{\mathrm{HeOH}, \mathrm{rt}, 20 \mathrm{~h}}^{\mathrm{O}}$

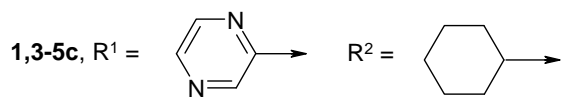

1, 3-5d, $\mathrm{R}^{1}=$
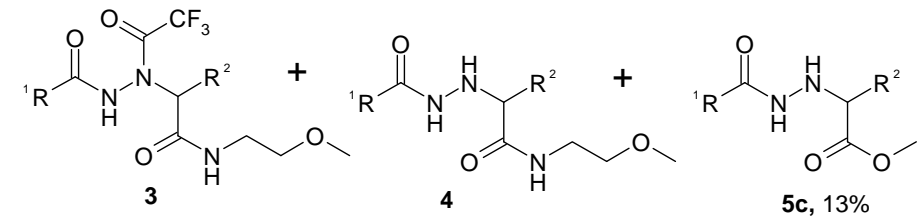

5d, $24 \%$ 
Scheme 3. Possible mechanistic rationale for the formation of the by-products $\mathbf{4}$ and $\mathbf{5}$ during the hydrazino-Ugi reaction in methanol.

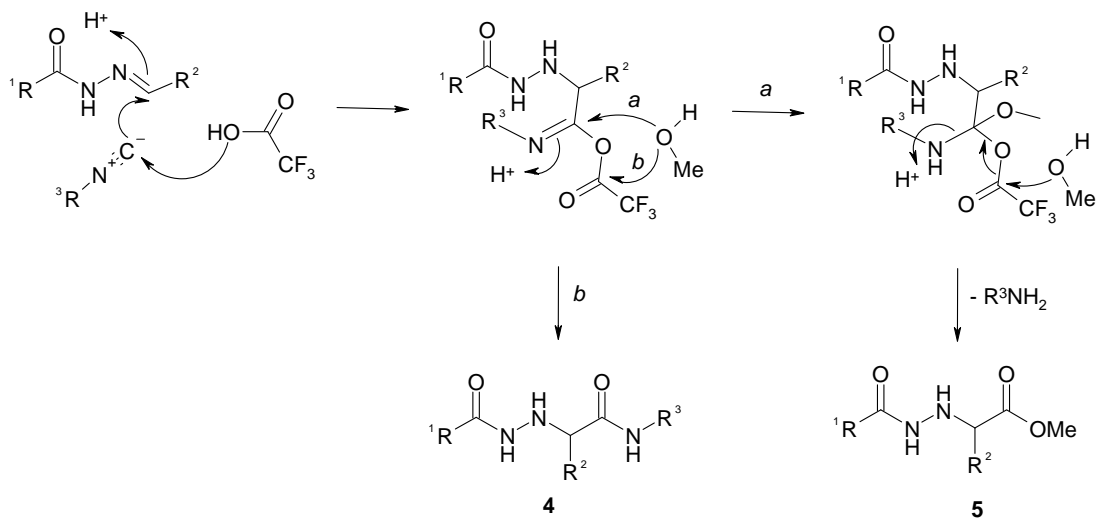

Scheme 4. Preparation of the hydrazone 6 and its 'intramolecular' hydrazino-Ugi reaction.

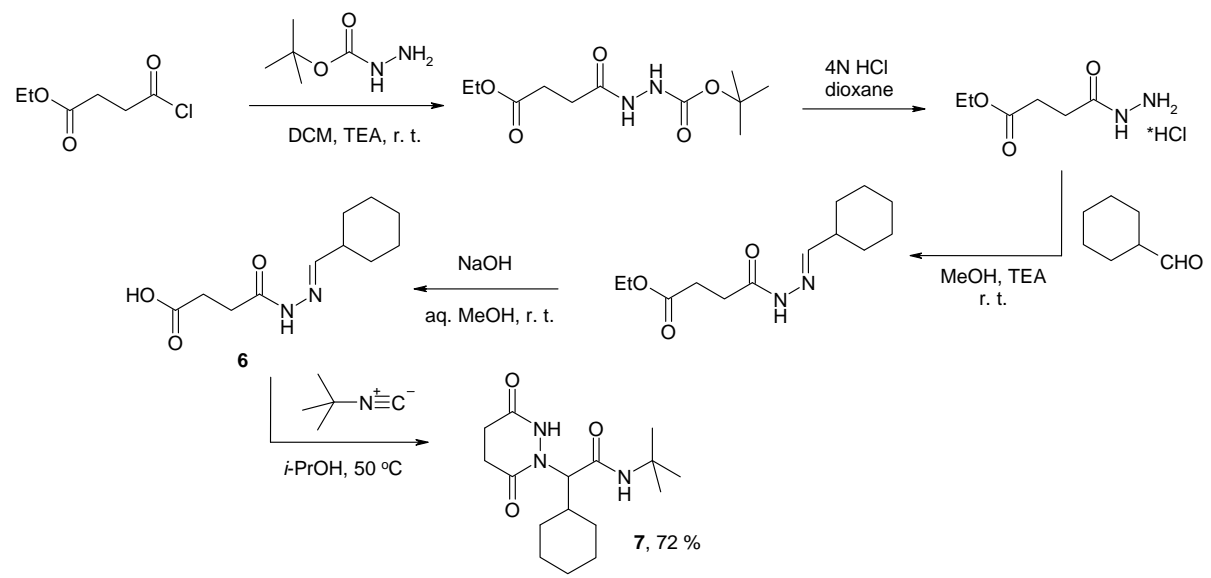

Scheme 5. Mechanistic rationale for the formation of the methyl ester 8 upon reaction of $\mathbf{6}$ with $t$-BuNC in methanol.

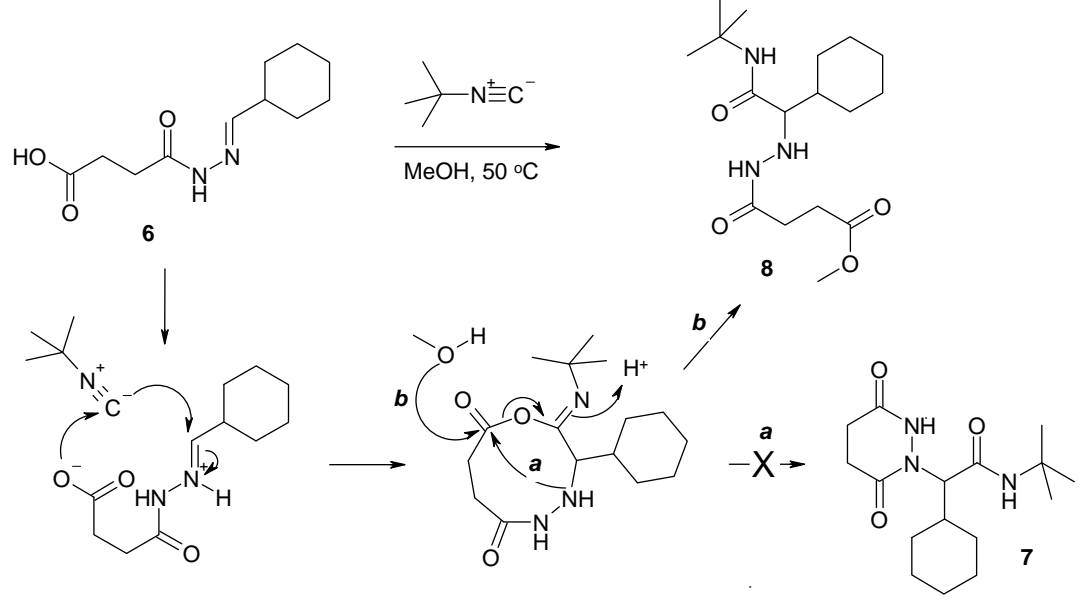

Scheme 6. Synthesis of $N^{(\alpha)}, N^{(\alpha)}$-bisalkyl, $N^{(\beta)}$-acyl hydrazines 9 via reductive alkylation. 


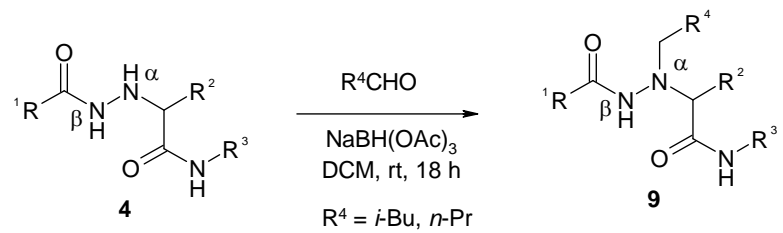

Table 1. $N^{(\alpha)}$-alkyl, $N^{(\beta)}$-acyl hydrazines 4 synthesized in this work.

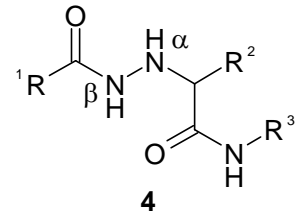

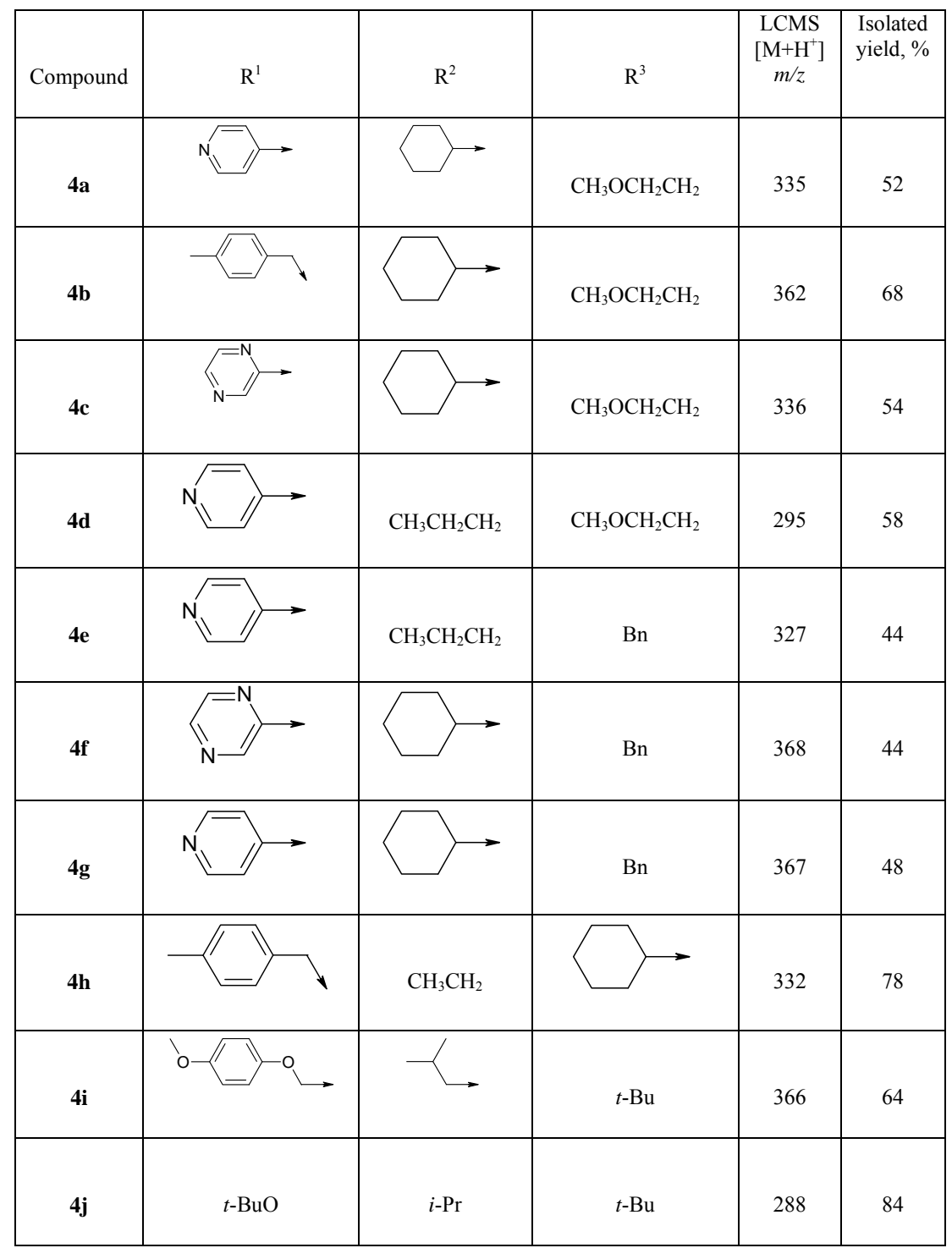




\begin{tabular}{|c|c|c|c|c|c|}
\hline $4 k$ & $t-\mathrm{BuO}$ & $i-\operatorname{Pr}$ & 1 & 352 & 84 \\
\hline 41 & $\mathrm{Me}$ & & & 334 & 77 \\
\hline $4 m$ & $\mathrm{Ph}$ & & $\mathrm{CH}_{3} \mathrm{OCH}_{2} \mathrm{CH}_{2}$ & 334 & 59 \\
\hline
\end{tabular}

Table 2. $N^{(\alpha)}, N^{(\alpha)}$-bisalkyl, $N^{(\beta)}$-acyl hydrazines 9 synthesized in this work.<smiles>[R]CN(NC([R])=O)C([R])C(=O)N[R]</smiles>

\begin{tabular}{|c|c|c|c|c|c|c|}
\hline Compound & $\mathrm{R}^{1}$ & $\mathrm{R}^{2}$ & $\mathrm{R}^{3}$ & $\mathrm{R}^{4}$ & $\begin{array}{c}\text { LCMS } \\
{\left[\mathrm{M}+\mathrm{H}^{+}\right]} \\
\mathrm{m} / \mathrm{z}\end{array}$ & $\begin{array}{l}\text { Isolated } \\
\text { yield, } \%\end{array}$ \\
\hline $9 a$ & & & $\mathrm{CH}_{3} \mathrm{OCH}_{2} \mathrm{CH}_{2}$ & $i-\mathrm{Bu}$ & 405 & 62 \\
\hline $9 b$ & & & $\mathrm{CH}_{3} \mathrm{OCH}_{2} \mathrm{CH}_{2}$ & $i-B u$ & 432 & 85 \\
\hline 9c & & & $\mathrm{CH}_{3} \mathrm{OCH}_{2} \mathrm{CH}_{2}$ & $n-\operatorname{Pr}$ & 392 & 58 \\
\hline 9d & & $\mathrm{CH}_{3} \mathrm{CH}_{2} \mathrm{CH}_{2}$ & $\mathrm{CH}_{3} \mathrm{OCH}_{2} \mathrm{CH}_{2}$ & $n-\operatorname{Pr}$ & 365 & 64 \\
\hline $9 e$ & & $\mathrm{CH}_{3} \mathrm{CH}_{2} \mathrm{CH}_{2}$ & $\mathrm{CH}_{3} \mathrm{OCH}_{2} \mathrm{CH}_{2}$ & $i-\mathrm{Bu}$ & 351 & 62 \\
\hline 9f & & & $\mathrm{Bn}$ & $n-\operatorname{Pr}$ & 438 & 74 \\
\hline $9 \mathrm{~g}$ & & & $\mathrm{Bn}$ & $n-\operatorname{Pr}$ & 424 & 72 \\
\hline 9h & & & $\mathrm{Bn}$ & $i-\mathrm{Bu}$ & 437 & 77 \\
\hline
\end{tabular}




\begin{tabular}{|c|c|c|c|c|c|c|}
\hline $\mathbf{9 i}$ & & & & & \\
\hline $\mathbf{9 j}$ & & $\mathrm{CH}_{3} \mathrm{CH}_{2} \mathrm{CH}_{2}$ & $t-\mathrm{Bu}$ & & 423 & 68 \\
\hline $\mathbf{9 k}$ & & $\mathrm{CH}_{3} \mathrm{CH}_{2} \mathrm{CH}_{2}$ & $\mathrm{Bn}$ & $n-\mathrm{Bu}$ & 397 & 80 \\
\hline
\end{tabular}

Table 3. Changes in ${ }^{1} \mathrm{H}$ NMR chemical shift values of the amide nitrogen-bound protons of hydrazinopeptide untis 4, 9 and the respective 'reference fragments' 10 and 11, upon solvent change (from $d_{6}$-DMSO to $\left.\mathrm{CDCl}_{3}\right)^{a}$

\begin{tabular}{|c|c|c|c|c|c|c|}
\hline \multirow{2}{*}{ Compound } & \multicolumn{3}{|c|}{$\Delta \delta\left(d_{6}-\mathrm{DMSO} \rightarrow \mathrm{CDCl}_{3}\right) \mathrm{H}^{1}$} & \multicolumn{3}{|c|}{$\Delta \delta\left(d_{6}-\mathrm{DMSO} \rightarrow \mathrm{CDCl}_{3}\right) \mathrm{H}^{2}$} \\
\hline & $4(9)$ & 10 & $\begin{array}{l}\text { Difference } \\
\{\mathbf{4}(\mathbf{9})-10\}\end{array}$ & $4(9)$ & 11 & $\begin{array}{l}\text { Difference } \\
\{\mathbf{4}(\mathbf{9})-\mathbf{1 1}\}\end{array}$ \\
\hline $4 a$ & 2.43 & 2.47 & 0.04 & 0.73 & 2.00 & 1.27 \\
\hline $4 b$ & 2.21 & 2.39 & 0.18 & 0.95 & 2.00 & 1.05 \\
\hline $4 \mathrm{c}$ & 1.56 & 1.29 & -0.27 & 0.49 & 2.00 & 1.51 \\
\hline 4d & 2.40 & 2.47 & 0.07 & 0.74 & 2.00 & 1.26 \\
\hline $4 e$ & 1.77 & 2.47 & 0.70 & 0.99 & 2.49 & 1.50 \\
\hline 4f & 0.87 & 1.29 & 0.42 & 0.88 & 2.69 & 1.81 \\
\hline $4 g$ & 2.17 & 2.47 & 0.30 & 0.99 & 2.69 & 1.70 \\
\hline $4 h$ & 2.53 & 2.39 & -0.14 & 1.02 & 2.09 & 1.07 \\
\hline $4 i$ & 1.08 & 1.49 & 0.41 & 0.50 & 1.99 & 1.49 \\
\hline $4 j$ & 2.09 & 1.79 & -0.30 & 0.51 & 2.04 & 1.53 \\
\hline $4 \mathbf{k}$ & 2.12 & 1.79 & -0.33 & 0.71 & 2.30 & 1.59 \\
\hline 41 & 1.65 & 1.51 & -0.14 & 0.71 & 2.09 & 1.38 \\
\hline $4 m$ & 1.50 & 2.09 & 0.59 & 0.58 & 2.00 & 1.42 \\
\hline $9 a$ & 0.24 & 2.47 & 2.23 & 2.48 & 2.00 & -0.48 \\
\hline $9 b$ & 0.89 & 2.39 & 1.50 & 1.98 & 2.00 & 0.02 \\
\hline 9c & 0.05 & 1.29 & 1.24 & 1.82 & 2.00 & 0.18 \\
\hline 9d & 0.42 & 2.47 & 2.05 & 1.81 & 2.00 & 0.19 \\
\hline $9 e$ & 0.40 & 2.47 & 2.07 & 1.73 & 2.00 & 0.27 \\
\hline 9f & 0.28 & 1.29 & 1.01 & 2.28 & 2.69 & 0.41 \\
\hline $9 g$ & 0.10 & 1.29 & 1.19 & 2.29 & 2.69 & 0.40 \\
\hline $9 \mathrm{~h}$ & -0.02 & 2.47 & 2.49 & 2.39 & 2.69 & 0.30 \\
\hline $9 i$ & 0.08 & 2.47 & 2.39 & 2.38 & 2.69 & 0.31 \\
\hline 9j & 0.24 & 2.47 & 2.23 & 2.17 & 2.49 & 0.32 \\
\hline 9k & 0.54 & 2.47 & 1.93 & 1.89 & 2.49 & 0.60 \\
\hline
\end{tabular}

${ }^{a}$ The ${ }^{1} \mathrm{H}$ NMR spectra of samples containing $15 \mu \mathrm{mol}$ of 4, 9, 10 or $\mathbf{1 1}$ in $0.6 \mathrm{~mL}$ of solvent were recorded at the temperature of $273 \mathrm{~K}$ throughout this study. 